\title{
Influência da variação de temperatura nos aspectos microestruturais e propriedades mecânicas no bronze de alumínio-níquel UNS C63000 submetido ao processo de têmpera
}

\author{
Vinicius Torres dos Santos ${ }^{1,2^{*}}$ (1) \\ Márcio Rodrigues da Silva ${ }^{2}$ \\ Huang Han Pang ${ }^{2}$ \\ Flávia Gonçalves Lobo ${ }^{2,3}$ \\ Givanildo Alves dos Santos ${ }^{4}$ \\ Wilson Carlos da Silva Junior ${ }^{4}$ \\ Antonio Augusto Couto ${ }^{1}$
}

\section{Resumo}

Tendo em vista o grande campo de aplicação industrial de ligas de bronze, torna-se indispensável a realização de estudos metalúrgicos detalhados buscando o desenvolvimento dessas ligas, especificamente bronze de alumínio-níquel, e uma das principais frentes tecnológicas em que o bronze está inserido é nas aplicações aeroespaciais, as quais exigem propriedades mecânicas específicas para que o material de engenharia suporte os esforços envolvidos. Uma das possibilidades do alcance dessas propriedades é por meio da imposição das variáveis presentes no tratamento térmico, o que influência na microestrutura, devido ao fato do bronze possuir transformação martensítica, representada pela fase $\beta$, e consequentemente no comportamento mecânico do material. O objetivo deste trabalho é mostrar a relação entre o endurecimento por têmpera, com posterior revenimento, e as propriedades mecânicas geradas no bronze aeroespacial UNS C63000. Os resultados mostram que temperaturas de aquecimento na ordem de 785,835 e $885^{\circ} \mathrm{C}$, seguidas de resfriamento brusco em água promovem a elevação dos resultados mecânicos, no entanto, nota-se a redução gradativa do percentual de fase $\alpha$.

Palavras-chave: UNS C63000; Têmpera; Microestrutura; Propriedades mecânicas.

\section{Influence of temperature variation on microstructural aspects and mechanical properties in nickel-aluminum bronze UNS C63000 submitted to the quenching process}

\begin{abstract}
In view of the large field of industrial application of bronze alloys, it becomes essential to carry out detailed metallurgical studies seeking the development of these alloys, specifically nickel-aluminum bronze, and one of the main technological fronts in which bronze is inserted is in aerospace applications, which require specific mechanical properties for the engineering material to support the efforts involved. One of the possibilities of reaching the properties is through the imposition of the variables present in the heat treatment, which influences the microstructure, due to the fact that bronze has martensitic transformation represented by $\beta$ phase, and, consequently, on the mechanical behavior of the material. The objective of this work is to show the relationship between hardening by quenching, with subsequent tempering, and the mechanical properties generated in the aerospace bronze UNS C63000. The results show that the score in the order of 785,835 and $885^{\circ} \mathrm{C}$ followed by sudden cooling in water promotes an increase in the mechanical results, however, it is noted a gradual reduction in the percentage of $\alpha$ phase.
\end{abstract}

Keywords: UNS C63000; Quenching; Microstructure; Mechanical properties.

${ }^{1}$ Universidade Presbiteriana Mackenzie, São Paulo, SP, Brasil.

${ }^{2}$ Termomecânica São Paulo S.A., São Bernardo do Campo, SP, Brasil.

${ }^{3}$ Universidade Federal do ABC, Santo André, SP, Brasil.

${ }^{4}$ Instituto Federal de Educação, Ciência e Tecnologia de São Paulo, São Paulo, SP, Brasil.

*Autor correspondente: vinicius.santos@termomecanica.com.br

2176-1523 C 2022. Santos et al. Publicado pela ABM. Este é um artigo publicado em acesso aberto (Open Access) sob a licença Creative Commons Attribution, que permite uso, distribuição e reprodução em qualquer meio, sem restrições desde que o trabalho original seja corretamente citado. 


\section{Introdução}

As ligas de bronze de alumínio com transformação martensítica surgiram em 1856 com o metalurgista inglês John Percy, o qual notou que uma pequena fração de alumínio elevava a dureza das ligas de cobre e, desde então, o alumínio vem sendo adicionado em proporções maiores na busca de propriedades mecânicas diferenciadas [1].

As ligas de bronze de alumínio são à base de cobre e podem apresentar percentuais de alumínio na ordem de até $14 \%$ em massa, porém, adições inferiores de níquel e ferro também são praticadas para proporcionar diferentes propriedades à liga, tais como resistência mecânica, ductilidade e resistência à corrosão [1-4].

Entre todas as ligas do grupo de bronze de alumínio, destaca-se o bronze de alumínio-níquel, também conhecido em ordem mundial como NAB (Nickel-Aluminium Bronze), contendo percentuais aproximados em massa de $80 \%$ de cobre, $10 \%$ de alumínio, $5 \%$ de níquel e $5 \%$ de ferro [5]. Ressaltando que a composição química do bronze aeroespacial UNS C63000, liga estudada neste trabalho, está próxima destes valores. As ligas de bronze de alumínio-níquel forjadas possuem aplicações no segmento militar aeronáutico na produção de buchas de eixos de trens de pouso (landing gear bushings), fazendo parte dos sistemas de articulações dos componentes aeroespaciais devido à combinação de resistência mecânica, tenacidade e resistência à corrosão $[1,6]$.

Em função da presença de diversos intermetálicos na microestrutura do bronze de alumínio-níquel, esse material é considerado como uma liga complexa $[3,5,7,8]$.
As fases do bronze de alumínio-níquel podem ser vistas no diagrama de fases do sistema cobre - alumínio com adição de $5 \%$ de níquel e $5 \%$ de ferro, ilustrado na Figura 1a. Considerando-se 10\% em massa de alumínio, podemos notar a presença de fases sólidas como alfa $(\alpha)$, beta $(\beta)$ e kappa $(\kappa)$, as quais apresentam morfologias e composições químicas distintas, interferindo, de forma consequente, nas propriedades mecânicas da liga $[1,9,10]$.

A Figura 1b ilustra o modelo do desenvolvimento da cristalização da liga de bronze de alumínio-níquel considerada, em que é possível notar que a partir da fase $\beta$ é gerada a fase $\alpha$ e também diversos intermetálicos denominados como kappa I (кI), kappa II (кII), kappa III (кIII) е kappa IV (кIV).

De forma direta, Brent [12] descreve os intermetálicos kappa's como sendo:

- Kappa I (кI): microconstituinte que se apresenta com formato de rosetas;

- Kappa II (кII): precipitado esferoidizado localizado preferencialmente no contorno do grão;

- Kappa III (кIII): fase lamelar oriunda de uma decomposição eutetoide; e

- Kappa IV (кIV):precipitado fino localizado dentro dos grãos de fase alfa.

A fase $\beta$ presente no início da cristalização dessa liga pode ficar retida na microestrutura, mesmo considerando a prática do resfriamento lento, e é considerada indesejável devido a sua susceptibilidade à corrosão preferencial, uma

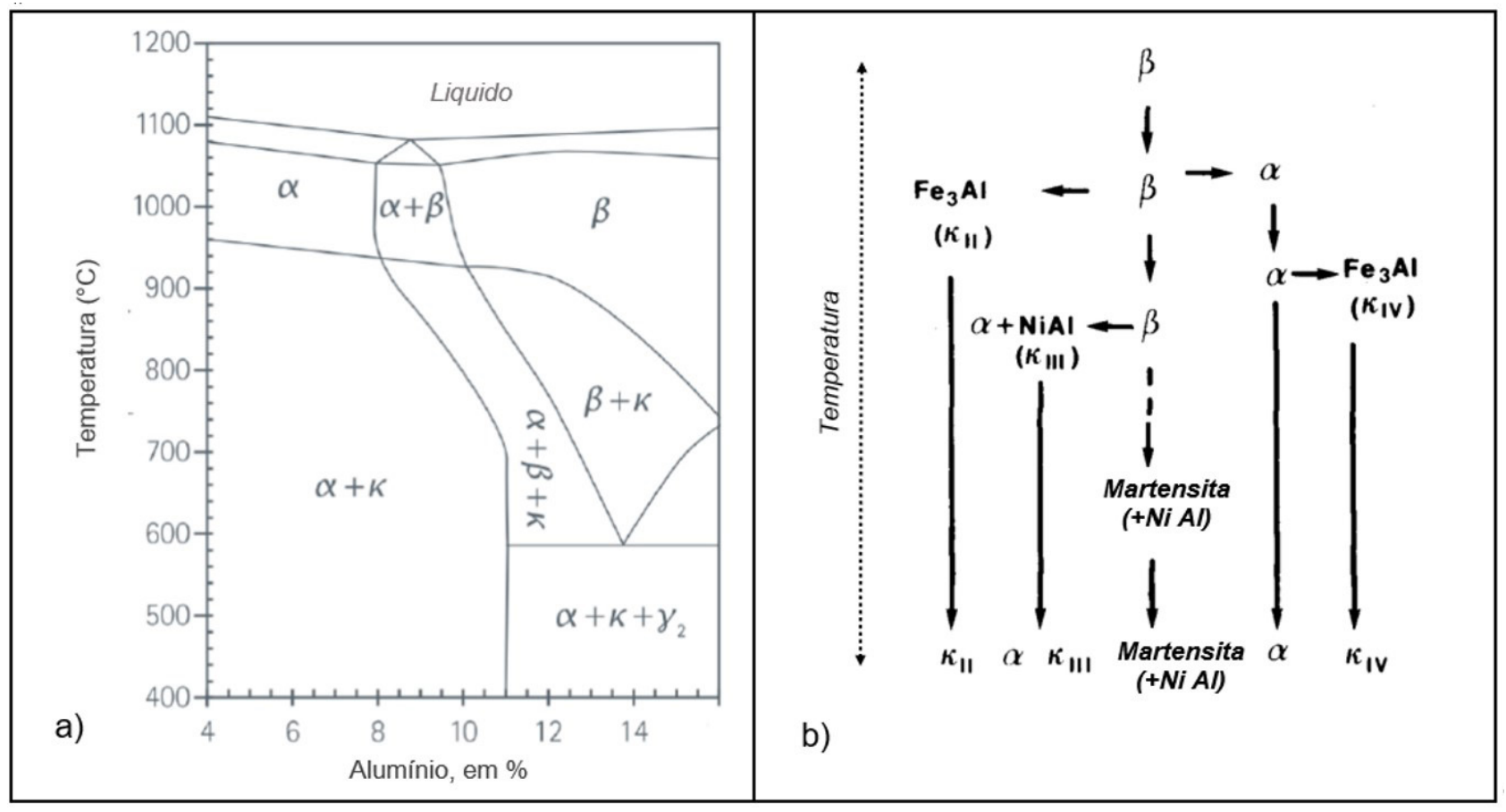

Figura 1. (a) Diagrama de fases pseudo-binário do sistema $\mathrm{Cu}-\mathrm{Al}$, com adição de $5 \%$ de níquel e $5 \%$ de ferro [11]; (b) Modelo de cristalização do bronze de alumínio-níquel [8]. 
vez que a fase $\beta$ retida transforma-se em fase martensítica metaestável, que é rica em partículas de $\mathrm{NiAl}$, o que aumenta a resistência mecânica e dureza da liga, porém necessita de tratamento térmico de revenimento para diminuir a fragilidade $[6,8,13,14]$.

Um estudo feito por Rodrigues et al. [6] demonstrou que na transformação martensítica, a velocidade de resfriamento é o que determina o nível de resistência mecânica do material, uma vez que seu aumento induz maior formação de fase $\beta$.

No caso do objetivo ser apenas um revenimento, recomenda-se fazer o tratamento térmico conforme procedimento da norma TQ50, visto que tal tratamento promove a decomposição eutetoide da martensita $\beta$ na fase $\alpha$ e no intermetálico KIII.

Quando o intermetálico kappa I está presente na microestrutura, ele apresenta-se em formatos de grandes rosetas e é rico em ferro e alumínio $\left(\mathrm{Fe}_{3} \mathrm{Al}\right)$. Entretanto, esse intermetálico está presente na microestrutura somente para composições químicas que apresentam percentuais acima de $5 \%$ em massa de ferro $[4,8-10,13,14]$.

A formação da fase $\beta$ é proveniente da reação peritética $(L+$ kappa $I \rightarrow \beta)$. No entanto, a fase kappa I é pouco discutida, pois está presente somente em faixas de temperaturas próximas da temperatura liquidus da liga [14].

A fase $\alpha$, ilustrada na Figura 2, é uma matriz sólida de equilíbrio com uma estrutura cristalina $\mathrm{CFC}$, e que se forma a partir da fase $\beta$, em torno de $1030^{\circ} \mathrm{C}$, exibindo uma estrutura com morfologia Widmanstätten $[8,9]$.

A fase $\alpha$ é dúctil e começa a aparecer na cristalização do bronze de alumínio- níquel aproximadamente a partir de $900{ }^{\circ} \mathrm{C}$, sendo que permanece na microestrutura até a temperatura ambiente [15].

Kappa III é um intermetálico localizado dentro da matriz $\alpha$, que se forma a partir da resultante da transformação eutetoide ( $\beta \rightarrow \alpha+$ Kappa III) e que ocorre em temperaturas próximas a $800^{\circ} \mathrm{C}[14]$. Entre todos os intermetálicos Kappa's presentes na liga, considera-se o Kappa III como o único precipitado kappa rico em níquel e alumínio (NiAl), e que tem a sua formação iniciada em temperaturas inferiores a $800{ }^{\circ} \mathrm{C}$.

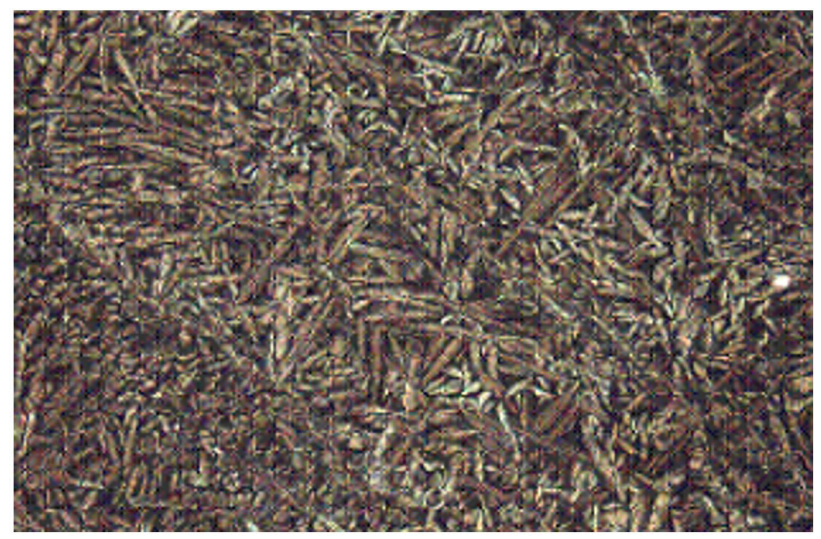

Figura 2. (a) Micrografia óptica do bronze de alumínio-níquel, apresentando morfologia Widmanstätten e com ampliação de 100x [1].
Kappa III pode apresentar-se com formato de lamelas em microestruturas de materiais resfriados lentamente. Essa fase é considerada como responsável pela ação anticorrosiva do NAB, sendo que, quanto maior o percentual em massa de níquel, maior a formação de kappa III $[1,3,8]$.

Kappa II consiste em precipitados na forma de pequenas rosetas, os quais ficam localizados no contorno do grão da fase $\alpha$ [1]. Os intermetálicos de Kappa II são ricos em ferro e alumínio ( $\left.\mathrm{Fe}_{3} \mathrm{Al}\right)$, e formam-se na mesma faixa de temperatura em que ocorre a formação de Widmanstätten $\alpha$, destacando que ambos são provenientes da fase $\beta[8,10]$.

As partículas de intermetálicos Kappa IV encontram-se distribuídas discretamente no seio da matriz $\alpha$ e apresentam-se na forma de precipitados finos globulares. As fases Kappa IV e Kappa II são ricas em ferro e alumínio $\left(\mathrm{Fe}_{3} \mathrm{Al}\right)$, portanto, para diferenciá-las, é preciso levar em consideração as suas respectivas morfologias $[3,8]$.

Por meio da análise das respectivas morfolologias presentes na Figura 3, Wharton et al. [16] identificaram todos os intermetálicos e fases presentes na microestrutura da liga de bronze de alumínio- níquel.

Essa norma descreve dois processos de fabricação distintos responsáveis pela obtenção das propriedades mecânicas finais desse material, as quais devem ser de 201 a $248 \mathrm{HB}$ para a dureza, de $758 \mathrm{MPa}$ como valor mínimo para o limite de resistência à tração, de $469 \mathrm{MPa}$ como valor

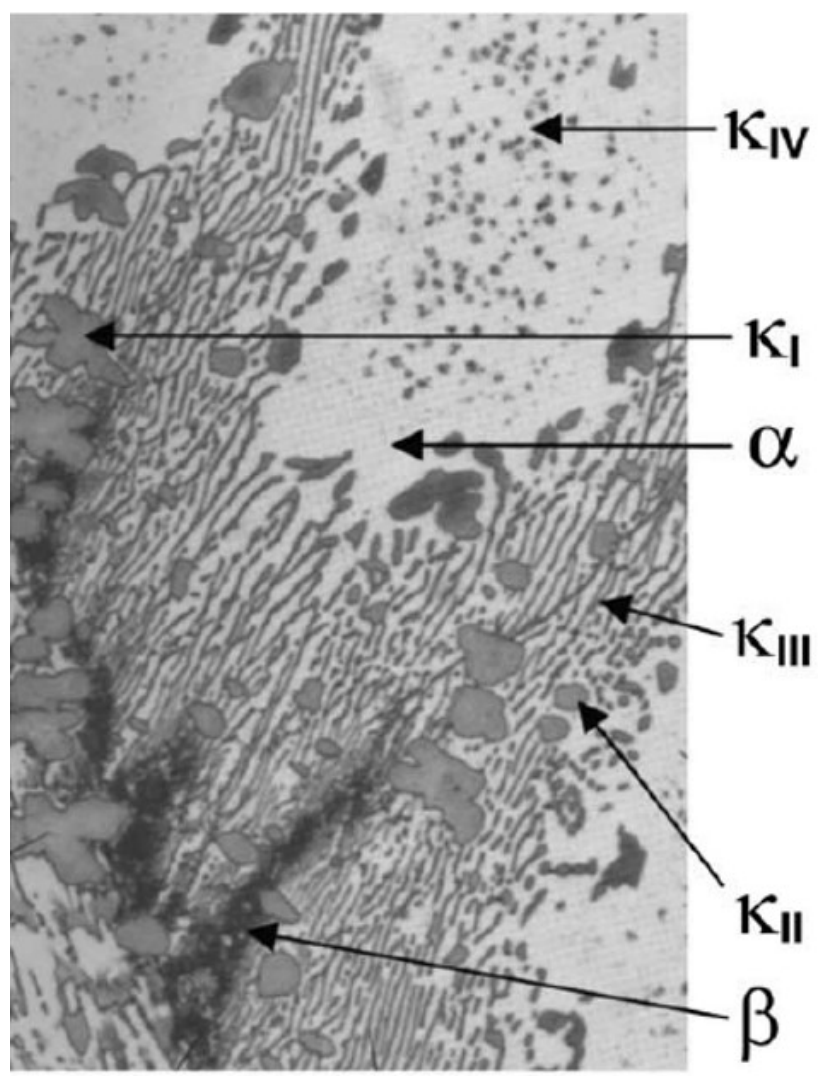

Figura 3. Microestrutura do bronze de alumínio-níquel obtida por microscopia eletrônica de varredura [16]. 
mínimo para o limite de escoamento a $0,5 \%$ de deformação padrão e com alongamento mínimo de $10 \%$.

Esses processos de fabricação são denominados TQ50 e HR50. O processo TQ50, conhecido como "quenching", é obtido por meio do processo de conformação a quente e, posteriormente, conta com o auxílio do aquecimento seguido do resfriamento brusco em água ou óleo para elevação de resistência mecânica e dureza e de posterior revenimento.

Para o processo HR50, conhecido também como "draw and stress relivied", as propriedades mecânicas são adquiridas somente pelo processo de conformação a frio (trefilação) com posterior processo de revenimento [5].

$\mathrm{O}$ fator motivador para a abordagem da verificação da influência dos parâmetros de tratamento térmico na microestrutura e propriedades mecânicas da liga de bronze de alumínio-níquel, considerando-se a têmpera TQ50, está fundamentado no fato de que o processo mais utilizado para fabricação desta liga é por meio de HR50, enquanto que o processo via TQ50 é pouco utilizado, sendo consequentemente o menos desenvolvido.

Considera-se também como fator motivador o estudo sobre as transformações de fases presentes no bronze de alumínio-níquel forjado e temperado e suas aplicações finais no segmento aeronáutico, o que torna tecnologicamente imprescindível a realização de pesquisas sobre o tratamento térmico para verificação do comportamento microestrutural e mecânico desse material.

O objetivo deste trabalho é mostrar a influência do endurecimento por têmpera, com posterior revenimento, na microestrutura e nas propriedades mecânicas geradas no bronze aeroespacial UNS C63000.

\section{Materiais e métodos}

Os materiais utilizados neste trabalho consistem em barras redondas extrudadas de bronze de alumínio-níquel, com 25,40 mm de seção transversal, cortadas em trechos de $300 \mathrm{~mm}$ de comprimento, sendo identificadas como B1, B2 e B3, cujas respectivas composições químicas são mostradas na Tabela 1 .

Para análise da composição química das amostras extrudadas, utilizou-se o equipamento de espectrometria fluorescente de raios X, modelo MAGIX FAST.

As amostras de barras redondas foram adquiridas por meio do processo de extrusão direta, proporcionando a redução da seção transversal do tarugo em 35 vezes. As amostras foram submetidas ao aquecimento por meio de um forno laboratorial do tipo mufla. Foram utilizadas três temperaturas distintas, sendo 785,835 e $885^{\circ} \mathrm{C}$ por 2 horas e, posteriormente, as amostras foram resfriadas bruscamente em água em temperatura ambiente. No que diz respeito aos parâmetros adotados no processo de revenimento, utilizou-se a temperatura de $650^{\circ} \mathrm{C}$ por 3 horas para todas as amostras. A caracterização mecânica foi realizada por teste de dureza em cinco pontos de cada amostra, em conformidade com a norma ASTM E10 [18], por meio de durômetro universal Wolpert com carga de $62,5 \mathrm{~kg}$ e esfera de $2,5 \mathrm{~mm}$. Os resultados de dureza apresentados em cada amostra referem-se a uma média de 5 medições. Foi utilizado também um equipamento de tração universal Instron, com capacidade de 100 kN, para obtenção dos valores de limites de resistência à tração e de escoamento e alongamento dos corpos de prova.

Os ensaios metalográficos foram realizados em conformidade com a norma ASTM E3 [19], adotando o sequenciamento da preparação superficial das amostras em lixas d'agua 200, 400 e 600 mesh e, posteriormente, foi realizado polimento com sucessiva imersão dos corpos de prova em uma solução metalográfica denominada como grade 7 por 25 segundos, a qual tem sua concentração composta por $10,5 \%$ em peso de ácido clorídrico e o restante de etanol. As amostras também foram analisadas por meio de microscopia

Tabela 1. Comparação da composição química padrão prevista na norma AMS 4640 [17] com a composição química das amostras B1, B2 e B3 utilizadas neste trabalho

\begin{tabular}{|c|c|c|c|c|c|}
\hline & \multicolumn{5}{|c|}{ Elementos (\% em massa) } \\
\hline & Al & $\mathbf{N i}$ & $\mathbf{F e}$ & Outros & $\mathrm{Cu}$ \\
\hline $\begin{array}{c}\text { AMS } 4640 \text { / UNS } \\
\text { C63000 [17] }\end{array}$ & 9,0 a 11 & 4,0 a 5,5 & 2 a 4 & $\begin{array}{l}\text { Mn - 1,5 máx. } \\
\mathrm{Zn} \text { - 0,30 máx. } \\
\mathrm{Sn} \text { - 0,20 máx. } \\
\mathrm{Si} \text { - 0,25 máx. }\end{array}$ & Restante \\
\hline B1 & 10,68 & 4,19 & 3,22 & $\begin{array}{c}\mathrm{Mn}-0,22 \\
\mathrm{Zn}-0,016 \\
\mathrm{Sn}-0,15 \\
\mathrm{Si}-0,19\end{array}$ & Restante \\
\hline B2 & 10,63 & 4,23 & 3,22 & $\begin{array}{c}\mathrm{Mn}-0,25 \\
\mathrm{Zn}-0,014 \\
\mathrm{Sn}-0,15 \\
\mathrm{Si}-0,13\end{array}$ & Restante \\
\hline B3 & 10,62 & 4,2 & 3,19 & $\begin{array}{c}\mathrm{Mn}-0,21 \\
\mathrm{Zn}-0,018 \\
\mathrm{Sn}-0,17 \\
\mathrm{Si}-0,14\end{array}$ & Restante \\
\hline
\end{tabular}


óptica no equipamento Leica, para verificação das morfologias da microestrutura, sendo que, para a avaliação da fração volumétrica da fase $\alpha$ utilizou-se o software Leica QPhase, em conformidade com a ASTM E1245 [20].

\section{Resultados e discussões}

As propriedades mecânicas encontradas nos corpos de prova antes da realização do experimento estão descritas na Tabela 2.

A Figura 4 refere-se à micrografia da amostra prensada obtida por meio de microscopia óptica, na qual se nota a fração volumétrica de fase $\alpha$ no percentual de $24,7 \%$ e microconstituintes intermetálicos dispersos na estrutura do material.

A partir das características mecânicas e microestruturais encontradas, as amostras foram submetidas ao aquecimento nas temperaturas e tempo descritos anteriormente. A Tabela 3 destaca os valores das propriedades mecânicas encontrados em cada amostra, em que foi feito o aquecimento seguido do resfriamento brusco na água em temperatura ambiente.

Os valores apresentados na Tabela 3 demonstraram que ocorreu a elevação do limite de resistência à tração, limite de escoamento e dureza, conforme a temperatura do tratamento térmico foi aumentada; em contrapartida, o alongamento diminuiu com esse aumento da temperatura, haja visto que, conforme o modelo de cristalização da liga de bronze de alumínio-níquel, mostrado na Figura 1b, a maior temperatura possibilita maior retenção da fase martensítica $\beta$, o que incrementa propriedades como resistência mecânica e dureza no material. Na Figura 5 são demonstradas as microscopias ópticas das amostras B1, B2 e B3, com os respectivos percentuais das frações volumétricas da fase $\alpha$ contida na microestrutura.

Nas amostras B1 e B2, nas quais foram utilizadas as temperaturas de tratamento térmico de 785 e $835^{\circ} \mathrm{C}$, respectivamente, observou-se a fase $\alpha$ de forma mais intensa, apresentando-se com formato circular e com a presença de microconstituintes Kappa's dispersos em toda microestrutura da amostra, enquanto que na amostra B3 $\left(885^{\circ} \mathrm{C}\right)$, a intensidade da fase $\alpha$ é menor, o que está de acordo com o modelo de cristalização do bronze de alumínio-níquel. Neste modelo, nota-se que a fase $\alpha \mathrm{em}$ temperaturas elevadas ainda está no início da decomposição, apresentando-se portanto, em menores frações volumétricas, uma vez que em temperaturas elevadas a predominância é da fase martensítica $\beta$, a qual fica retida na microestrutura no processo de têmpera, necessitando de revenimento para

Tabela 2. Propriedades mecânicas das amostras extrudadas

\begin{tabular}{ccccc}
\hline & $\begin{array}{c}\text { Limite de resistência à } \\
\text { tração } \mathbf{( M P a )}\end{array}$ & $\begin{array}{c}\text { Limite de escoamento à } \\
\text { deformação padrão de } \\
\mathbf{0 , 5 \%}(\mathbf{M P a})\end{array}$ & Alongamento (\%) & Dureza (HB) \\
\hline B1 & 795 & 380 & 15 & 195 \\
B2 & 796 & 384 & 14 & 197 \\
B3 & 793 & 379 & 17 & 194 \\
\hline
\end{tabular}

Tabela 3. Resultados das propriedades mecânicas obtidas após o aquecimento seguido de resfriamento brusco em água

\begin{tabular}{cccccc}
\hline & $\begin{array}{c}\text { Temperatura e } \\
\text { tempo do tratamento } \\
\text { térmico }\end{array}$ & $\begin{array}{c}\text { Limite de resistência } \\
\text { à tração (MPa) }\end{array}$ & $\begin{array}{c}\text { Limite de escoamento } \\
\text { à deformação padrão } \\
\text { de } \mathbf{0 , 5} \text { (MPa) }\end{array}$ & Alongamento (\%) & Dureza (HB) \\
\hline $\mathrm{B} 1$ & $785^{\circ} \mathrm{C}-2 \mathrm{~h}$ & 839 & 348 & 10 & 215 \\
$\mathrm{~B} 2$ & $835^{\circ} \mathrm{C}-2 \mathrm{~h}$ & 842 & 381 & 6,5 & 242 \\
$\mathrm{~B} 3$ & $885^{\circ} \mathrm{C}-2 \mathrm{~h}$ & 883 & 440 & 5 & 255 \\
\hline
\end{tabular}

(a)

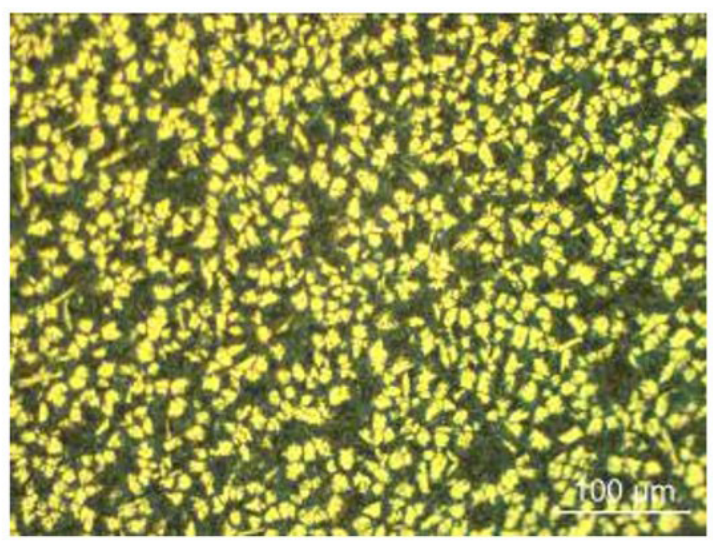

(b)

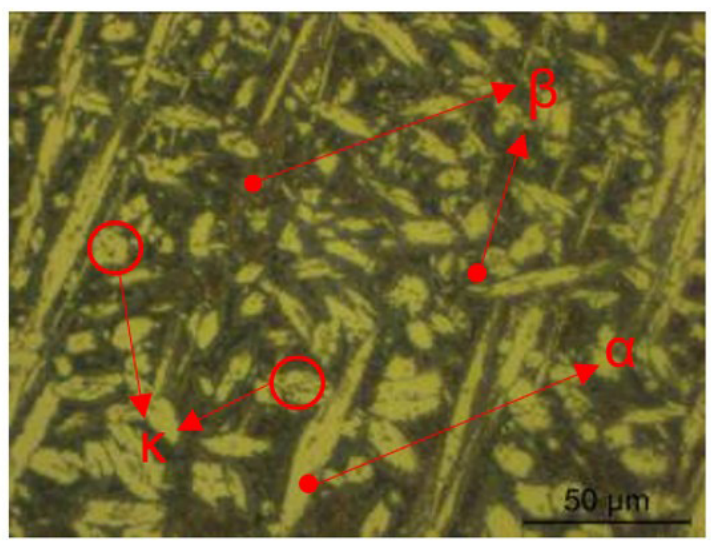

Figura 4. (a) Micrografia da microestrutura da amostra extrudada (200x); (b) Micrografia da microestrutura da amostra extrudada com detalhamento das fases e intermetálicos Kappa's (500x). 
(a)

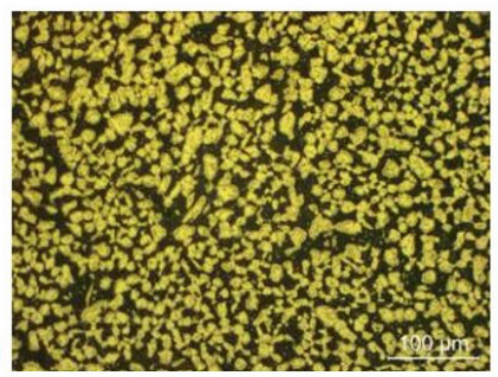

(b)

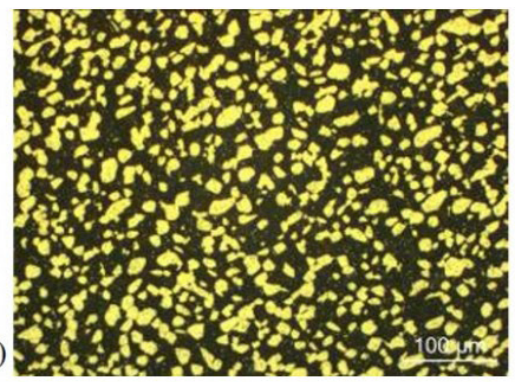

(c)

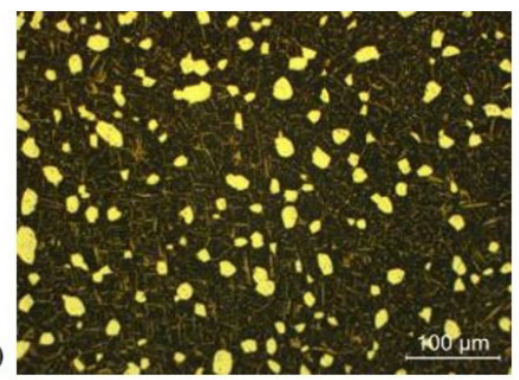

Figura 5. (a) Micrografia da microestrutura da amostra B1 com fração volumétrica de $42 \%$ de fase $\alpha$ (200x); (b) Micrografia da microestrutura da amostra B2 com fração volumétrica de 29,6\% de fase $\alpha$ (200x); e (c)Micrografia da microestrutura da amostra B3 com fração volumétrica de $21 \%$ de fase $\alpha(200 x)$.

Tabela 4. Propriedades mecânicas das amostras após o processo de revenimento

\begin{tabular}{|c|c|c|c|c|c|c|}
\hline & $\begin{array}{l}\text { Temperatura } \\
\text { do tratamento } \\
\text { térmico }\end{array}$ & Revenimento & $\begin{array}{l}\text { Limite de } \\
\text { resistência à tração } \\
\text { (MPa) }\end{array}$ & $\begin{array}{c}\text { Limite de } \\
\text { escoamento à } \\
\text { deformação padrão } \\
\text { de } 0,5 \%(\mathrm{MPa}) \\
\end{array}$ & Alongamento (\%) & Dureza (HB) \\
\hline B1 & $785^{\circ} \mathrm{C}-2 \mathrm{~h}$ & $650^{\circ} \mathrm{C}-4 \mathrm{~h}$ & 747 & 417 & 18 & 183 \\
\hline B2 & $835^{\circ} \mathrm{C}-2 \mathrm{~h}$ & $650{ }^{\circ} \mathrm{C}-4 \mathrm{~h}$ & 780 & 445 & 16 & 205 \\
\hline B3 & $885^{\circ} \mathrm{C}-2 \mathrm{~h}$ & $650{ }^{\circ} \mathrm{C}-4 \mathrm{~h}$ & 820 & 534 & 15 & 210 \\
\hline
\end{tabular}

que ela seja decomposta em $\alpha+\kappa$ III (reação eutetoide), o que reduz naturalmente os valores de limite de resistência à tração, limite de escoamento e dureza.

Na Tabela 4 são demonstrados os resultados das propriedades mecânicas das amostras após o processo de revenimento, realizado a $650{ }^{\circ} \mathrm{C}$ por 4 horas em todas as amostras.

Por meio da análise dos resultados da Tabela 4, nota-se que a amostra B3 é a mais efetiva no atendimento aos valores de propriedades mecânicas previstos na norma AMS 4640 (2011). Ressaltando que na amostra B3 foi adotado o parâmetro de $885^{\circ} \mathrm{C}$ por 2 horas para realização da têmpera e posterior revenimento de $650{ }^{\circ} \mathrm{C}$ por 4 horas.

\section{Conclusão}

Observou-se a elevação do limite de resistência à tração, limite de escoamento e dureza de todas as amostras ensaiadas conforme a temperatura do tratamento térmico foi sendo aumentada. Após o revenimento, ocorreu a redução das propriedades avaliadas, sendo possível notar que somente a amostra B3 atendeu aos requisitos da norma AMS 4640. $\mathrm{O}$ alongamento apresentou desempenho inversamente proporcional às demais propriedades como já era esperado.

A elevação do limite de resistência à tração, limite de escoamento, dureza e a redução notável do alongamento estão fundamentadas no fato de que quanto maior a variação de temperatura entre a liga de bronze de alumínio-níquel e a solução na qual ocorre o resfriamento severo, maior a retenção da fase martensítica $\beta$ (CCC), que é rica em $\mathrm{NiAl}$ e, consequentemente, menor a intensidade da fase $\alpha$ (CFC). Dessa forma, o processo de revenimento torna-se indispensável para o material.

Após o processo de têmpera a amostra B1 tratada termicamente a $785{ }^{\circ} \mathrm{C}$ apresentou $42 \%$ de fração volumétrica de fase $\alpha$, devido ao fato de que a esta temperatura, no modelo de cristalização, a fase $\alpha$ já está formada na microestrutura. Por outro lado, a amostra B3, por ter sido tratada termicamente a $885^{\circ} \mathrm{C}$, em que a fase $\alpha$ ainda está iniciando a sua formação, notou-se na microestrutura, fração volumétrica menor (21\%) em relação à amostra $\mathrm{B} 1$.

\section{Agradecimentos}

Os autores agradecem à Dra. Regina Celi Venâncio, em nome do conselho curador da Fundação Salvador Arena, ao Chief Executive Officer da Termomecanica São Paulo $\mathrm{S} / \mathrm{A}$ Sr. Luiz Henrique Caveagna, em nome de todo o time técnico, pela disponibilização da estrutura para confecção das amostras e testes laboratoriais e ao Instituto Federal de São Paulo e à Universidade Presbiteriana Mackenzie, pela parceria na realização de pesquisas em ligas de cobre.

\section{Referências}

1 Richardson I. Guide to nickel aluminum bronze for engineers. London: Copper Alliance; 2016. (Copper Development Association; no. 222) 
2 Vaidyanath LR. The manufacture of aluminium-bronze casting. Calcutta: Indian Copper Information Center; 1968.

3 Culpan EA, Rose G. Microstructural characterization of cast nickel aluminium bronze. Journal of Materials Science. 1978;13:1647-1656.

4 Nascimento MS, Santos GA, Teram R, Santos VT, Silva MR, Couto AA. Effects of thermal variables of solidification on the microstructure, hardness, and microhardness of Cu-Al-Ni-Fe alloys. Materials. 2019;12:1267.

5 Meigh HJ. Cast and wrought aluminum bronzes properties-processes and structure. 2nd ed. London: Iom Communucations; 2000.

6 Rodrigues CA, Melo MLNM, Paes LES. Caracterização de uma liga de bronze de alumínio submetida a diferentes tratamentos térmicos. Metals and Materials. 2012;65(3):343-348.

7 Weston GM. Survey of nickel-aluminum bronze casting alloys for marine applications. Melbourne: Materials Research Laboratories; 1981. (Australia Department of Defence Report DSTO MRL-R807).

8 Ahanafrooz A, Hasan F, Lorimer GW, Ridley N. Microstructural development in complex nickel-aluminum bronze. Metallurgical Transactions A. 1983;14(10):1951-1956.

9 Faires KB. Characterization of microstructure and microtexture in longitudinal sections from friction stir processed Nickel-Aluminum Bronze [thesis]. Monterey: Naval Postgraduate School; 2003.

10 Pierce FA. The Isothermal deformation of nickel aluminum bronze in relation to friction stir processing [thesis]. Monterey: Naval Postgraduate School; 2004.

11 Sláma P, Dlouhy J, Kover M. Influence of heat treatment on the microstructure and mechanical properties of aluminium bronze. Materials Technology. 2014;48(4):599-604.

12 Brent KB. Characterization of microstructure and microtexture in longitudinal sections from friction stir processed nickel-aluminum bronze [thesis]. Monterey: Naval Postgraduate; 2003.

13 Hasan F, Jahanafrooz A, Lorimer GW, Ridley N. The morphology, crystallography, and chemistry of phases in as-cast nickel-aluminum bronze. Metallurgical Transactions A. 1982;13a:1337-1345.

14 Howell PR. On the phases, microconstituents and microstructures in nickel-aluminum bronze. McLean: Department of Materials Science and Engineering; 2000 [acesso em 24 Abr. 2021]. Disponível em: http://www.copper.org/ publications/pub_list/pdf/A1310-Microstructures-NickelAlumBronzes.pdf

15 Pisarek B. Model of Cu-Al-Fe-Ni bronze crystallization. Archives of Foundry Engineering. 2013;13(3):72-79.

16 Wharton JA, Barik RC, Kear G, Wood RJK, Stokes KR, Walsh FC. The corrosion of nickel-aluminium bronze in seawater. Corrosion Science. 2005;47:3336-3367.

17 Specification AM. AMS 4640: aluminum bronze, bars, rods, shapes, tubes, and forgings $81,5 \mathrm{Cu}-10,0 \mathrm{Al}-4,8 \mathrm{Ni}$ -3,0Fe - Draw and Stress Relieved (HR50) or Temper Annealed (TQ50). Warrendale: SAE Internacional Group; 2011.

18 American Standard Test Methods. ASTM E10: standard test method for brinell hardness of metallic materials. West Conshochen: ASTM; 2012.

19 American Standard Test Methods. ASTM E3: preparation of metallographic specimens. West Conshochen: ASTM; 2012

20 American Standard Test Methods. ASTM E1245: standard practice for determining the inclusion or second-phase constituent content of metals by automatic image analysis. West Conshochen: ASTM; 2008.

Recebido em: 20 Nov. 2020

Aceito em: 24 Abr. 2021 